\title{
SYNTHETIC PHOTOMETRY
}

Chairman and Editor : R. BUSER

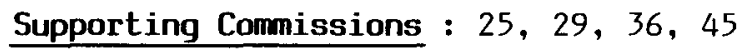




\title{
PRINCIPLES AND SCOPE OF SYNTHETIC PHOTOMETRY
}

\author{
Roland Buser \\ Astronomical Institute, University of Basel, Switzerland \\ and \\ Space Telescope Science Institute, Baltimore, U.S.A.
}

ABSTRACT. We describe the synthetic photometry algorithm and its general usefulness in a wide range of applications.

\section{INTRODUCTION}

Synthetic photometry refers to an algorithm which provides magnitudes and colors computed from a spectral energy flux distribution and the response functions of a photometric system. In our era of powerful computers, the simplicity of this algorithm may be disappointing, but its generality is significant: since there are very many-real as well as imaginary-response functions, flux distributions, and photometric data, respectively, there is a tremendous number of ways in which synthetic photometry is able to produce useful results.

For example, one might wish to extrapolate the color-temperature relation to cooler and higher-luminosity stars by fitting the observed result with the purely theoretical relation calculated from a grid of model atmosphere flux distributions. One might also like to predict the metallicity dependence of a newly designed photometric index by computing the colors from the laboratory measurements of the instrumental response functions and a set of globular cluster star flux scans covering a range in $[\mathrm{Fe} / \mathrm{H}]$. Or one might even have to generate, just from available photoelectric UBV data, a magnitude sequence of faint standard stars for calibrating a photographic near-IR survey, and one would choose to compute the magnitudes for both photometric systems from a library of stellar spectrophotometric data suitable to derive the required transformations.

These few applications also illustrate how synthetic photometry is essential in probing or discovering the connections between different, and even widely separated, pieces of information. Can we already hope to develop these into the coherent knowledge that we presume to extend from the physics of the stellar atmospheres to the colors of faint galaxies? How do the errors propagating along affect our views of these more or less distant horizons? How reliable are, to begin with, the basic ingredients of the synthetic photometry algorithm? 
While these questions are important to many astronomers, it is up to the specialists in stellar classification, photometry, spectroscopy, and atmospheres to provide the initial answers. Therefore, the present meeting was designed to initiate a first-hand discussion of the backbones of synthetic photometry, viz., the observed and the theoretical stellar energy distributions, as well as the passbands of the photometric systems. This outline of the principles and scope of synthetic photometry is meant to put the more scrutinizing discussion into perspective.

\section{PRINCIPLES OF SYNTHETIC PHOTOMETRY}

\subsection{Numerical Algorithm}

The basic equation is the definition of the mean flux density, which makes a precise connection between the flux spectrum and the flux measurement that is made by a (broad-band) photometric detector:

$$
f_{\lambda}\left(P_{i}\right) \equiv \int_{0}^{\infty} f_{\lambda}(\lambda) P_{i}(\lambda) d \lambda / \int_{0}^{\infty} P_{i}(\lambda) d \lambda .
$$

Here $f_{\lambda}(\lambda)$ is the energy flux density distribution of the source, in erg $\mathrm{cm}^{-2} \mathrm{~s}^{-1}$ $A^{-1}$, and $P_{i}(\lambda)$ is the dimensionless response function of the photometric passband $i$. The function $P_{i}(\lambda)$ is actually the product of the characteristic functions of all the individual components in the optical path of the instrument, including the reflection efficiencies of the mirrors, the transmission efficiencies of the filter(s), and the quantum efficiency of the detector.

Equation (1) assigns weights proportional to the energy of each detected photon (i.e., $f_{\lambda}(\lambda)$ ), which is appropriate for synthesizing measurements obtained by an instrument that does so, too. In the more recent years, however, photon-counting instruments have become widespread. With these techniques, the flux density integrated over a finite passband must be proportional to the observed photon countrate; hence the mean flux density must be defined in such a way that equal weight is assigned to each detected photon. Conversion of $f_{\lambda}(\lambda)$ to a photon flux thus changes the basic equation (1) to

$$
f_{\lambda}\left(P_{i}\right) \equiv \int_{0}^{\infty} f_{\lambda}(\lambda) \lambda P_{i}(\lambda) d \lambda / \int_{o}^{\infty} \lambda P_{i}(\lambda) d \lambda .
$$

Equations (1) and (2) are evaluated by numerical integration of the flux distribution over the passband function, both of which must be known with sufficient resolution at a sufficiently large number of wavelength points. Synthetic magnitudes are then simply calculated as

$$
m_{i}=-2.5 \log f_{\lambda}\left(P_{i}\right)+k_{i}
$$

where $k_{i}=$ constant defines the zero-point for passband $i$. Of course, for a multicolor system, like for example, the Johnson UBV, the synthetic color indices may then be computed as

$$
c_{i j}=m_{i}-m_{j}
$$


The difference between equations (1) and (2) results in differences of up to several hundredths of a magnitude in the predicted UBV data of cool stars $\left(T_{e f f}<5000\right.$ $\mathrm{K})$. For hotter stars, these differences are in general smaller $(<0.02 \mathrm{mag}$ ), for the magnitudes as well as the color indices. In comparing computed and observed data, it is thus necessary to verify if pulse-counting instruments were used in the observations, and to choose the appropriate equation for the synthetic calculations.

\subsection{Passband Evaluation}

For any given photometric system, synthetic photometry should first be used to establish that the observed photometric data are consistent with the observed spectrophotometric data of the stars. It is imperative to determine those passband functions, $P_{i}(\lambda)$, which yield synthetic magnitudes matching the observed ones, for the determination of the zero-point constants, $k_{i}$. Thus, the linear regressions

$$
m_{i} \text { (observed) }=m_{i} \text { (synthetic), }
$$

and consequently,

$$
c_{i j} \text { (observed) }=c_{i j} \text { (synthetic), }
$$

must be verified for the whole magnitude and color ranges covered by the photometric observations. This can be done using, for example, the existing catalogs of observed stellar energy distributions discussed by Hayes (1986, this volume). If the synthesized magnitudes and colors deviate systematically from the observations (e.g., if scale factors different from unity, or nonlinearities occur in the regressions (5) and/or (6); see, e.g., Buser 1978), they should be used to derive the appropriate passband functions by least-squares (e.g., Rufener and Maeder 1971, see also Rufener 1986, this volume) or maximum entropy methods (Horne 1985).

For any photometric system that is well-defined observationally, such deviations in general indicate that the passband functions used for the synthetic calculations do not match those behind the observations. Even passband functions that were measured in the laboratory and tabulated in the literature may have wavelengths, widths, and/or shapes different from those which ensure that the stellar photometric and spectrophotometric observations are consistent. Unless this basic requirement is met, however, the photometric observations can neither be used for conclusively testing the synthetic photometry of theoretical model atmospheres - which would really also jeopardize any direct theoretical calibration and interpretation of the observed data in terms of the physical stellar atmospheric parameters.

The Johnson UBV system provides an illustrative example. Following the principles described above, the UBV passbands were evaluated (Buser 1978) using the library of stellar spectrophotometric data published by Straizys and Sviderskiene (1972). The resulting response functions take account of the fact (Johnson 1963) that the standard $U-B$ colors include residual atmospheric extinction, while the $B-V$ colors are truly extraatmospheric. These same response functions were also used successfully in reproducing the observed stellar colors for the Gunn and Stryker (1983) spectrophotometric atlas (Labhardt and Buser 1985). As a result, the adopted 
UBV response functions represent the mean observed two-color loci with high accuracy over the complete ranges covered by population I stars of all $\mathrm{MK}$ types and luminosity classes. Consequently, they were combined with various grids of theoretical model atmospheres for DA white dwarf, G-K giant, and O-G stars to compute synthetic colors (Buser and Kurucz 1978, 1985, 1986). The results appear to indicate that these theoretical energy distributions can be used with profit in synthetic photometry studies of a wide variety of passbands and stellar types, even though the theoreticians behind them have little doubts about why and how to improve their products significantly in the future (see Kurucz 1986, Gustafsson 1986 in this volume).

\section{THE SCOPE OF SYNTHETIC PHOTOMETRY}

Once the passbands of the photometric system(s) have been evaluated, the synthetic photometry algorithm can be used as a sort of universal translator providing the communication channels between different research areas. Essentially, all the available information associated with the spectral energy flux distributions put into equations (1) or (2) is also made available and can also be associated with the magnitudes and colors computed for whatever photometric system(s) we choose to investigate. The basic equations (1) or (2) of the algorithm can also be made more general by including additional separate functions that may determine the photometric measurement of the flux, such as the transmissions of the terrestrial atmosphere or the interstellar medium.

I shall depict this multi-directional flow of information by tracking its way just along the one line of sight from our knowledge of the stars to our ignorance of the universe.

\section{Stellar Evolution: Time}

The most fundamental step is taken from the stellar evolution theory to the photometric observations of the stars. Here, synthetic photometry of theoretical model atmospheres translates evolutionary tracks and isochrones from the $\left(M_{b o l}, T_{e f f}\right)$ plane to the color-absolute magnitude diagrams by providing bolometric corrections and colors as functions of the physical parameters. Application to clusters has allowed us to know the evolutionary stages and even the ages behind the stellar variety (e.g., VandenBergh 1983, 1985).

\section{Stellar Populations in the Galaxy: Space}

Another important step consists in using our detailed knowledge of the stellar populations in the solar neighborhood to analyze multicolor photometric survey data of more distant stars. Here, synthetic photometry translates the stellar luminosity functions, $\phi_{p}\left(M_{V}\right)$, into the survey-specific functions $\phi_{p}\left(M_{S}\right)$ by providing the luminosity- and metallicity-dependent transformations between the UBV and the survey photometric systems. Application to faint star counts and color distribu- 
tions in many galactic fields has refined our picture of the large-scale structure of the Galaxy (e.g., Buser and Kaeser 1985, Bahcall et al. 1985).

Evolution of Galaxies: Space-Time

The boldest step reaches for the galaxies as evolving systems of evolving populations of evolving stars. The libraries of observed and theoretical stellar flux distributions are used to generate evolutionary sequences of synthetic spectra for galaxies of different morphological types with different assumed histories of the star formation rate and/or initial stellar mass functions. Subsequently, synthetic photometry translates these spectra into the redshifted colors and faint magnitudes that one would expect to observe from large distances. Application to deep multicolor surveys of galaxies has indeed provided a promising look into the universe (e.g., Bruzual and Kron 1980, Koo 1985).

\section{Acknowledgements}

I am grateful to Keith Horne for stimulating discussions and to Dorothy Schlogel for help in preparing the manuscript. This work was supported by the National Aeronautics and Space Administration during my stay in Baltimore, and by the Swiss National Science Foundation.

\section{REFERENCES}

Bahcall, J.N., Ratnatunga, K.U., Buser, R., Fenkart, R.P., and Spaenhauer, A. 1985, Astrophys. J. 299, 616.

Bruzual A., G. and Kron, R.G. 1980, Astrophys. J. 241, 25.

Buser, R. 1978, Astron. Astrophys. 62, 411.

Buser, R. and Kurucz, R.L. 1978, Astron. Astrophys. 70, 555.

Buser, R. and Kaeser, U. 1985, Astron. Astrophys. 145, 1.

Buser, R. and Kurucz, R.L. 1985, in IAU Symposium No. 111: Calibration of Fundamental Stellar Quantities, D.S. Hayes, L.E. Pasinetti, A.G. Davis Philip eds., (Dordrecht: Reidel), p. 513.

Buser, R. and Kurucz, R.L. 1986, in preparation.

Gunn, J.E. and Stryker, L.L. 1983, Astrophys. J. Suppl. 52, 121.

Gustafsson, B. 1986, this volume.

Hayes, D.S. 1986, “Observed Stellar Energy Distributions For Synthetic Photometry", this volume.

Horne, K. 1985, private communication.

Johnson, H.L. 1963, in Basic Astronomical Data, ed. K. Aa. Strand, (Chicago: Univ. of Chicago Press), p. 204.

Koo, D.C. 1985, Astron. J. 90, 418.

Kurucz, R.L. 1986, Theoretical Stellar Energy Distributions, this volume. 
Labhardt, L. and Buser, R. 1985, in IAU Symposium No. 111: Calibration of Fundamental Stellar Quantities, D.S. Hayes, L.E. Pasinetti, A.G. Davis Philip eds., (Dordrecht: Reidel), p. 519.

Rufener, F. and Maeder, A. 1971, Astron. Astrophys. Suppl. 4, 43.

Rufener, F. 1986, "Passbands and Photometric Systems", this volume.

Straizys, V. and Sviderskiene, Z. 1972, Bull. Vilnius Astron. Obs. 35.

VandenBergh, D. 1983, Astrophys. J. Suppl. 51, 29.

VandenBergh, D. 1985, Astrophys. J. Suppl. 58, 711. 